\title{
Molecular characterization of the hexose transporter gene in benznidazole resistant and susceptible populations of Trypanosoma cruzi
}

\author{
Paula F dos Santos ${ }^{1}$, Jerônimo C Ruiz', Rodrigo P P Soares ${ }^{1}$, Douglas S Moreira', Antônio M Rezende ${ }^{1}$,
} Edson L Folador ${ }^{1}$, Guilherme Oliveira ${ }^{1,2,3}$, Alvaro J Romanha ${ }^{1}$ and Silvane M F Murta ${ }^{{ }^{*}}$

\begin{abstract}
Background: Hexose transporters (HT) are membrane proteins involved in the uptake of energy-supplying glucose and other hexoses into the cell. Previous studies employing the Differential Display technique have shown that the transcription level of the HT gene from T. cruzi (TcrHT) is higher in an in vitro-induced benznidazole (BZ)-resistant population of the parasite (17 LER) than in its susceptible counterpart (17 WTS).

Methods: In the present study, TcrHT has been characterized in populations and strains of $T$. cruzi that are resistant or susceptible to BZ. We investigated the copy number and chromosomal location of the gene, the levels of TcrHT mRNA and of TcrHT activity, and the phylogenetic relationship between TcrHT and HTs from other organisms.

Results: In silico analyses revealed that 15 sequences of the TcrHT gene are present in the T. cruzi genome, considering both CL Brener haplotypes. Southern blot analyses confirmed that the gene is present as a multicopy tandem array and indicated a nucleotide sequence polymorphism associated to T. cruzi group I or II. Karyotype analyses revealed that TcrHT is located in two chromosomal bands varying in size from 1.85 to $2.6 \mathrm{Mb}$ depending on the strain of T. cruzi. The sequence of amino acids in the HT from T. cruzi is closely related to the HT sequences of Leishmania species according to phylogenetic analysis. Northern blot and quantitative real-time reverse transcriptase polymerase chain reaction analyses revealed that TcrHT transcripts are 2.6-fold higher in the resistant 17 LER population than in the susceptible 17 WTS. Interestingly, the hexose transporter activity was $40 \%$ lower in the 17 LER population than in all other T. cruzi samples analyzed. This phenotype was detected only in the in vitro-induced BZ resistant population, but not in the in vivo-selected or naturally BZ resistant T. cruzi samples. Sequencing analysis revealed that the amino acid sequences of the TcrHT from 17WTS and 17LER populations are identical. This result suggests that the difference in glucose transport between 17WTS and 17LER populations is not due to point mutations, but probably due to lower protein expression level.
\end{abstract}

Conclusion: The BZ resistant population 17 LER presents a decrease in glucose uptake in response to drug pressure.

Keywords: Trypanosoma cruzi, Drug resistance, Hexose transporters

\footnotetext{
* Correspondence: silvane@cpqrr.fiocruz.br

${ }^{1}$ Centro de Pesquisas René Rachou/FIOCRUZ, Avenida Augusto de Lima

1715, Belo Horizonte 30190-002, MG, Brazil

Full list of author information is available at the end of the article
} 


\section{Background}

The kinetoplastid protozoan Trypanosoma cruzi is the causative agent of Chagas disease (American trypanosomiasis), the pathogen, vector and clinical characteristics of which were first described by Carlos Chagas in 1909. The disease currently affects $10-13$ million people in Latin America and is believed to have been responsible for the deaths of more than 10,000 in 2008 [1].

The drugs nifurtimox (NFX; 5-nitrofuran-(3-methyl-4(5' - nitrofurfurylideneamine) tetrahydro-4 H-1, 4-tiazine-1, 1-dioxide); Bayer] and benznidazole [BZ; 2-nitroimidazole (N-benzyl-2-nitroimidazole acetamide; Roche] are the only medications presently available for the treatment of Chagas disease, and both were developed empirically some 40 years ago. There are a number of issues associated with the use of these drugs, including the low percentage cure rate in the chronic phase (8\%) compared with that in the acute phase (76\%) [2], the age-dependent efficacy [3,4], and the undesirable side effects [5]. Another factor for concern is the appearance of parasite populations that are naturally resistant to NFX or BZ, and some with cross-resistance to both drugs [6-9]. The problems associated with the available drugs, and the lack of alternative medications, highlight the urgent need to develop new strategies for chemotherapy against Chagas disease [10].

One attractive approach to the identification of potential therapeutic targets is to focus on genes that are differentially expressed in strains of $T$. cruzi that are resistant or susceptible to NFX or BZ. In order to pursue this strategy, and with the additional objective of understanding the molecular basis of drug resistance, we have previously investigated the levels of gene expression in BZ resistant and susceptible $T$. cruzi populations using Differential Display (DD) and Representation of Differential Expression (RDE) techniques [11]. The T. cruzi hexose transporter gene ( $T c r H T)$ was one of four genes indicated by DD as being over-expressed in an in vitroinduced BZ resistant population (17 LER) of the protozoan. This gene was first characterized by Tetaud et al. [12], and comprises an open reading frame (ORF) of 1,635 bp encoding for 544 amino acids with a predicted mass of $60 \mathrm{kDa}$. The TcrHT protein belongs to the Major Facilitative Superfamily 1 (GLUT 1) of glucose transporters [13,14], comprises 12 transmembrane domains and is responsible for the cellular uptake of all energysupplying glucose and other hexoses. Once inside the cell, the hexose is directed to a highly specific kinetoplastid organelle, the glycosome, which contains the enzymes involved in glucose and glycerol metabolism. Various researchers have proposed hexose transporters (HTs) as potential drug targets by virtue of their critical role in glucose metabolism in the parasite or because hexose deprivation could affect the virulence of the parasite [15-17]. In addition, TcrHT has the capacity to transport D-fructose with a higher affinity than mammalian HTs, thus increasing the chances of being able to develop a more specific drug [18].

The aims of the present study were to characterize the TcrHT gene in populations and strains of T. cruzi that were either resistant or susceptible to BZ, and to establish the copy number and chromosomal location of the gene, the levels of TcrHT mRNA and of TcrHT activity, and the phylogenetic relationship between TcrHT and HTs from other organisms.

\section{Methods}

\section{Populations and strains of Trypanosoma cruzi}

The provenances of the T. cruzi population with in vivo selected BZ resistance (BZR) and its susceptible pair (BZS), and of the pair of BZR and BZS clones (16R and 4 S, respectively), have been reported previously [19]. The BZ-resistant $T$. cruzi population (17 LER) derived from the Tehuantepec cl2 susceptible wild-type strain (17 WTS) [20] was obtained in vitro by increasing in a stepwise manner the concentration of BZ. The 17 LER parasites are resistant to a dose of BZ 23 times higher than that required to kill $50 \%$ of the 17WTS parasites. These parasites were kindly provided by Dr. Philippe Nirdé (Génétique Moleculaire des Parasites et des Vecteurs, Montpellier, France). The three naturally resistant T. cruzi strains Colombiana, Yuyu and SC-28, and the susceptible strain CL have been characterized previously $[6,7]$. All of the populations and strains employed were classified within $T$. cruzi groups I to VI according to the nomenclature of Zingales et al. [21], and their epimastigote forms were maintained in liquid liver infusion tryptose (LIT) medium at $28^{\circ} \mathrm{C}$ [22].

\section{In silico and phylogenetic analyses of the TcrHT gene}

Similarity searches using the Basic Local Alignment Search Tool (BLAST; National Center for Biotechnology Information) (http://blast.ncbi.nlm.nih.gov/) were carried out between the TcrHT sequence (GenBank accession no. $\underline{\mathrm{U} 05588}$ ), and the non-redundant database (nr - NCBI). Based on this analysis, we were able to recover the PFAM profile PF00083, which is an evolutionary model of "sugar transporter protein". Subsequently, using this PFAM model, we used the hmmsearch tool [23] to search for protein sequences related to the model in the predicted proteomes of T. cruzi CL Brener Esmeraldo-like and nonEsmeraldo-like [24] from TriTrypDB (http://tritrypdb.org/ common/downloads/release-4.1/Tcruzi/). The hmmsearch returned the ID of those proteins related to PFAM model. Each positive BLAST hit was analyzed and the result of the alignment was compared with information available in the TriTrypDB database. In this study proteins were used that had an E-value less than $1 \mathrm{e}^{-7}$. 
In order to carry out a phylogenetic analysis, the sequence of TcrHT (GenBank accession no. U05588 or TritrypDB Tc00.1047053506355-10) from T. cruzi CL Brener non-Esmeraldo like predicted proteome was compared against the proteomes of T. cruzi CL Brener Esmeraldo like, two Trypanosoma spp. (T. congolense and T. brucei), four species of Leishmania (L. braziliensis, L. infantum, L. major and L. mexicana) and Trichomonas vaginalis as outgroup. For each proteome analyzed, the sequence with the highest similarity to Tc00.1047053506355.10 was used in the phylogenetic analysis. Initially, the sequences were aligned using the MAFFT software [25]. It was used in local multiple alignment mode which is suitable for analysis of a set of proteins that possess isolated domains. The alignment generated was trimmed using TrimAl [26] to select blocks of conserved regions. This step guarantees a better alignment quality. In order to choose the evolutionary model which best fits with this alignment, three models (JTT, Dayhoff and Blosum62) were tested using the ProtTest version 2.4 [27]. The best model according to ProtTest was JTT [28], and it was used in the analysis. All other phylogenetic analysis steps were performed using the PHYLIP package version 3.67 [29]. The first program used from the package was Seqboot that generated 1000 alignments from the TrimAl alignment output. Subsequently, ProML was used along with "analyze multiple dataset" option activated. This program uses Maximum Likelihood as phylogenetic method. Using the ProML program 1000 trees were generated. To extract a consensus tree the program Consense also from PHYLIP package was used. At the end of this process, a phylogeny in NEXUS format was generated, and it was read in FigTree version 1.2.3 software (http://tree.bio.ed.ac.uk/software/figtree), which is a tree figure drawing tool.

\section{Extraction and preparation of RNA and DNA}

The extraction and preparation of total RNA and genomic DNA from T. cruzi populations, together with the subsequent electrophoretic analyses, were carried out according to previously described procedures [30]. For the analyses of TcrHT genes, aliquots of genomic DNA $(14 \mu \mathrm{g})$ from each of the $T$. cruzi populations and strains were digested with the restriction endonucleases SalI and EcoRI (Invitrogen, Carlsbad, CA, USA). Southern and Northern blots were hybridized with ${ }^{32} \mathrm{P}$ labeled TcrHT probes according to the protocol of Murta et al. [31].

\section{Polymerase chain reaction (PCR)}

In order to prepare the probes employed in Southern and Northern blot assays, a 450 bp segment corresponding to nucleotide 273 to 723 of the TcrHT (GenBank accession no. U05588) was amplified from T. cruzi Y strain
DNA by PCR using the forward primer $5^{\prime}$ ATGCCATCCAAGAAGCAGACTGAT $3{ }^{\prime}$ and the reverse primer $5^{\prime}$ CTGCCCGGCATAGATCGACCCAATC 3'. Amplification was carried out in a Perkin Elmer (Waltham, MA, USA) GeneAmp 9600 thermocycler with a reaction mixture containing 0.5 Units of Taq DNA polymerase (Invitrogen), $1.5 \mathrm{mM} \mathrm{MgCl}_{2}, 200 \mathrm{mM}$ of each dNTP, $20 \mathrm{pmol}$ of each primer, $1 \mathrm{ng}$ of T. cruzi DNA and $1 \mathrm{X}$ specific Taq DNA polymerase buffer to a final volume of $10 \mu \mathrm{L}$. The PCR program comprised an initial denaturation at $95^{\circ} \mathrm{C}$ for $5 \mathrm{~min}, 30$ cycles of denaturation at $95^{\circ} \mathrm{C}$ for $1 \mathrm{~min}$, annealing at $65^{\circ} \mathrm{C}$ for $1 \mathrm{~min}$ and extension at $72^{\circ} \mathrm{C}$ for $1 \mathrm{~min}$, and a final extension at $72^{\circ} \mathrm{C}$ for $5 \mathrm{~min}$. The PCR product was subjected to electrophoresis on a $6 \%$ nondenaturing polyacrylamide gel.

\section{Real-time reverse transcriptase (RT)-PCR}

The protocol used for the preparation of first strand cDNA and the procedure for real-time RT-PCR were as previously described [30]. An ABI Prism 7000 - Sequence Detection System SDS (PE Applied Biosystems, Foster City, CA, USA) was employed in the quantitative realtime RT-PCR amplification of first strand cDNA using the specific primers 5' AGTTCCTTCACGTGGACG 3' (forward) and 5' CTGTGCCTTTTCTACGCT 3'(reverse) designed from the complete nucleotide sequence of TcrHT (GenBank accession no. U05588). The T. cruzi housekeeping gene hypoxanthine-guanine phosphoribosyltransferase (TcHGPRT), a T. cruzi single copy gene, was employed to normalize the amount of each sample assayed [31]. It is expressed at equivalent levels in all $T$. cruzi samples analyzed presenting a Cq (Quantitation cycle) of $22 \pm 0.78$. Standard curves were prepared for each run using known quantities of pCR 2.1-TOPO plasmids (Invitrogen) containing TcrHT and TcHGPRT genes.

\section{Pulsed-field gel electrophoresis (PFGE)}

Chromosomes from different T. cruzi populations and strains were separated by PFGE on an Amersham Pharmacia (GE Life Sciences, Little Chalfont, UK) Gene Navigator TM system as described previously [31]. Optimized separations for TcrHT were obtained using PFGE pulse intervals of: $250 \mathrm{~s}$ for $24 \mathrm{~h}, 500 \mathrm{~s}$ for $24 \mathrm{~h}, 750 \mathrm{~s}$ for $24 \mathrm{~h}$ and $1000 \mathrm{~s}$ for $24 \mathrm{~h}$ at $90 \mathrm{~V}$ and $9^{\circ} \mathrm{C}$. Following electrophoresis, gels were stained with ethidium bromide $(0.5 \mu \mathrm{g} / \mathrm{mL})$ and the chromosome bands were transferred onto nylon membranes and incubated with ${ }^{32} \mathrm{P}$ labeled TcrHT probe in order to identify the gene.

\section{Determination of TcrHT activity}

The method of Eisenthal et al. [32] was used, with slight modification, in order to assay the activity of TcrHT in epimastigote forms of the populations and strains of 
T. cruzi in the exponential phase of growth. Briefly, a sample of cells $\left(2 \times 10^{5}\right)$ was washed three times in Krebs-Ringer phosphate (KRP) medium ( $\mathrm{pH}$ 8.0) at room temperature for $5 \mathrm{~min}$ at $2,000 \times \mathrm{g}$, resuspended in KRP to a final volume of $100 \mu \mathrm{L}$, and transferred to a separate test tube. An aliquot $(5 \mu \mathrm{L})$ of KRP containing $0.6 \mu \mathrm{Ci}$ of 6-deoxy-D-[6- $\left.{ }^{3} \mathrm{H}\right]$ glucose (specific activity $11 \mathrm{Ci} / \mathrm{mmol}$ or $66.7 \mathrm{mCi} / \mathrm{mg}$ ) was added to each of the tubes and, after a time interval of 30,60 or $120 \mathrm{~s}$, the T. cruzi HTs were blocked by the addition of $200 \mu \mathrm{L}$ of $2 \mathrm{mM}$ phloridizin (Sigma, Saint Louis, MO, USA). In order to obtain an accurate estimate of TcrHT at $0 \mathrm{~s}$, the cells representing this time interval were added to a tube after the glucose and the phloridizin. Negative controls contained cells and phloridizin, while positive controls contained only radioactive glucose. After each time interval, the tubes containing the reactions were centrifuged at $4^{\circ} \mathrm{C}$ for $1 \mathrm{~min}$ at $10,000 \times \mathrm{g}$, and the supernatants containing glucose that had not been incorporated into the cells were discarded. Parasites were lyzed after resuspending in $500 \mu \mathrm{L}$ of deionized water, and radioactivity was measured on a Beckman Coulter (Brea, CA, USA) model LS liquid scintillation counter. The amount of glucose taken up by a cell sample was estimated on the basis that $1 \mathrm{~mol}$ of 6-deoxy-D-[6-3 H] glucose exhibited an activity of $9.26 \mathrm{e}^{+15} \mathrm{cpm}$ (determined by directly counting a known amount of the radioactive substrate). For each population or strain of $T$. cruzi, the total amount of protein present in $2 \times 10^{5}$ cells was quantified using the standard Bradford assay, and the amount of glucose transported per mg of total protein was then calculated as a function of time. Two-way analysis of variance (ANOVA) was used to compare conjointly the uptake variables, namely, amount of glucose and time. Data were analyzed using Minitab Statistical Software (Minitab Inc., State College, PA, USA) at the level of significance corresponding to $\alpha=0.05$.

\section{DNA sequencing}

The TcrHT 1,635 bp ORF from T. cruzi BZ-susceptible and -resistant populations (17WTS, 17LER, BZS and BZR) was cloned into the TOPO PCR2.1 vector (Invitrogen) and amplified in E. coli TOP 10 F' competent cells. Minipreparations of plasmid DNA were done using the QIAprep Spin Miniprep kit (Qiagen). Aliquots of $500 \mathrm{ng}$ DNA were sequenced using the DYEnamic ${ }^{\circledR}$ ET Dye Terminator Kit (GE Healthcare) in a MegaBACE $1000^{\circledR}$ DNA Analysis System (GE Healthcare), using the following primers: M13 forward 5'-GTAAAACGACGGCCAG-3', M13 reverse 5'-CAGGAAACAGCTATGAC-3' and internal TcrHT forward primers $5^{\prime}$-GCTCGTCCTAT AACGGC-3', 5' -CTGGAACTGACTGGCATC-3' , 5' 'TTGAGATTGGCCTTGGAC-3' and reverse primers 5' -ATTGGGTAGCAGACGTTG-3', 5' -AAAGTTCCAC GCCATCAC-3', 5' -AACGACACCTTGTGACCA-3' and
5'-GCATTCCCCATGTAT-3'. Reaction consisted of an initial denaturation at $95^{\circ} \mathrm{C}$ followed by 30 cycles of $15 \mathrm{~s}$ at $95^{\circ} \mathrm{C}, 20 \mathrm{~s}$ at $55^{\circ} \mathrm{C}$ and $80 \mathrm{~s}$ at $60^{\circ} \mathrm{C}$. Samples were analysed on Mega Bace 400 sequencer (Amersham) and the data were analysed using Phred, Phrap and Consed. Sequence variability between parasites was assessed by sequencing three colonies of each $T$. cruzi population and by sequencing each colony twice with each primer. Sequences selected for analysis were those with Phred > 40. Nucleotide sequences were translated into the amino acid sequence using Transec. The nucleotide and amino acid sequences were aligned using the ClustalW 2.1 software.

\section{Densitometric analyses}

Southern and Northern autoradiograms were photographed and subsequently analyzed using ImageMaster VDS software (GE Life Sciences, Little Chalfont, UK). T. cruzi $24 \mathrm{~S}$ alpha small subunit was used as loading control and for normalization of densitometric analysis in Northern blot assays. Differences were considered significant when the intensity band ratios were $\geq 2.0$.

\section{Results}

\section{In silico analyses of TcrHT gene}

A similarity search of the TcrHT sequence showed that 15 sequences within T. cruzi CL Brener Esmeraldo-like (TcChr-S - 7 sequences) and non-Esmeraldo-like (TcChr$\mathrm{P}-8$ sequences) matched the search criteria (Additional file 1: Table S1). Among these sequences, nine sequences were annotated as hexose/sugar transporter proteins, being four complete copies (Tc00. 1047053508551.30, Tc00.1047053511041.40, Tc00.1047053505183.130, Tc00. 1047053506355.10), three incomplete (Tc00. 1047053508551. 39, Tc00.1047053424937.10 and Tc00.1047053508231.9) and two truncated (Tc00.1047053504125.100 and Tc00. 1047053506355.100). Seven of these copies were located on chromosome TcChr37 of the Esmeraldo-like and nonEsmeraldo like CL Brener haplotypes and two on the chromosome TcChr26 of both haplotypes. Taking into account, the four complete sequences, we observed that three (Tc00.1047053508551.30, Tc00.1047053511041.40, Tc00.1047053506355.10) were compatible to the previously described TcrHT (Tetaud et al., 1994) and were aligned using ClustalW 2.1 software (Additional file 1: Figure S1). Although the sequence Tc00. 1047053505183. 130 presents 12 transmembrane domains and it has been annotated as a putative sugar transporter, it shows only $18 \%$ identity and $33 \%$ similarity when compared to the reference sequence Tc00.1047053506355.10 (GenBank accession no. U05588), thus it was not included our alignment analysis. The remaining six sequences were annotated as hypothetical proteins, however, they present the PFAM profile PF00083 and motif of "putative transporter 
protein". They are located on chromosome TcChr13 or TcChr39 of the Esmeraldo-like and non-Esmeraldo like CL Brener haplotypes (Additional file 1: Table S1).

It is worth mentioning that the non-completion of some of the sequences are due to their location in the contig or to the size of the contig used in the genome assemble. In the first case, the sequence started at the end of the contig, which terminated before the gene sequence was completed. In the second case, the contig itself was smaller than the gene sequence. Based on these findings as applied to version 2010-10-20 of the T. cruzi genome, we can only speculate that this situation is a function of the automatic annotation process adopted, and/or the incomplete annotation of the genome, and/or the highly repetitive content of the genome [33] considering that $c a .50 \%$ of the parasite genome consists of repetitive sequences [24].

In order to compare the amino acid sequence of TcrHT with sequences of HTs identified in different trypanosomatids, a maximum likelihood phylogenetic tree was constructed (Figure 1). The HT of T. cruzi (Esmeraldo like and non-Esmeraldo like haplotypes) was more closely related to the Leishmania species studied (namely, L. major, L. infantum, L. mexicana and $L$. braziliensis) than to other species of Trypanosoma (i.e. $T$. brucei and T. congolense). The very low identity (23\%) and similarity (37\%) observed between the sugar transporters of T. cruzi and Homo sapiens (GLUT 1) reinforces the potential of TcrHT as a target for chemotherapy against Chagas disease.

\section{Levels of TcrHT mRNA in T. cruzi populations}

Hybridization of the Northern blots of total RNA from T. cruzi samples against a specific $\left[{ }^{32} \mathrm{P}\right] \mathrm{dCTP}$ labeled TcrHT probe revealed one transcript of $2.6 \mathrm{~kb}$ for the T. cruzi group I population with in vitro-induced resistance (17 LER) and for its susceptible counterpart (17 WTS). However, an additional transcript of $2.1 \mathrm{~kb}$ was detected for the T. cruzi group II population with in vivo-selected resistance (BZR) and for its susceptible counterpart (BZS) (Figure 2A). In each case, a ribosomal RNA probe was used as a quantitative control (Figure 2B). Comparison of the results of densitometric analyses of transcript profiles of the populations (data not shown) revealed that the levels of TcrHT mRNA were 2 -fold greater in the drug-resistant 17 LER population

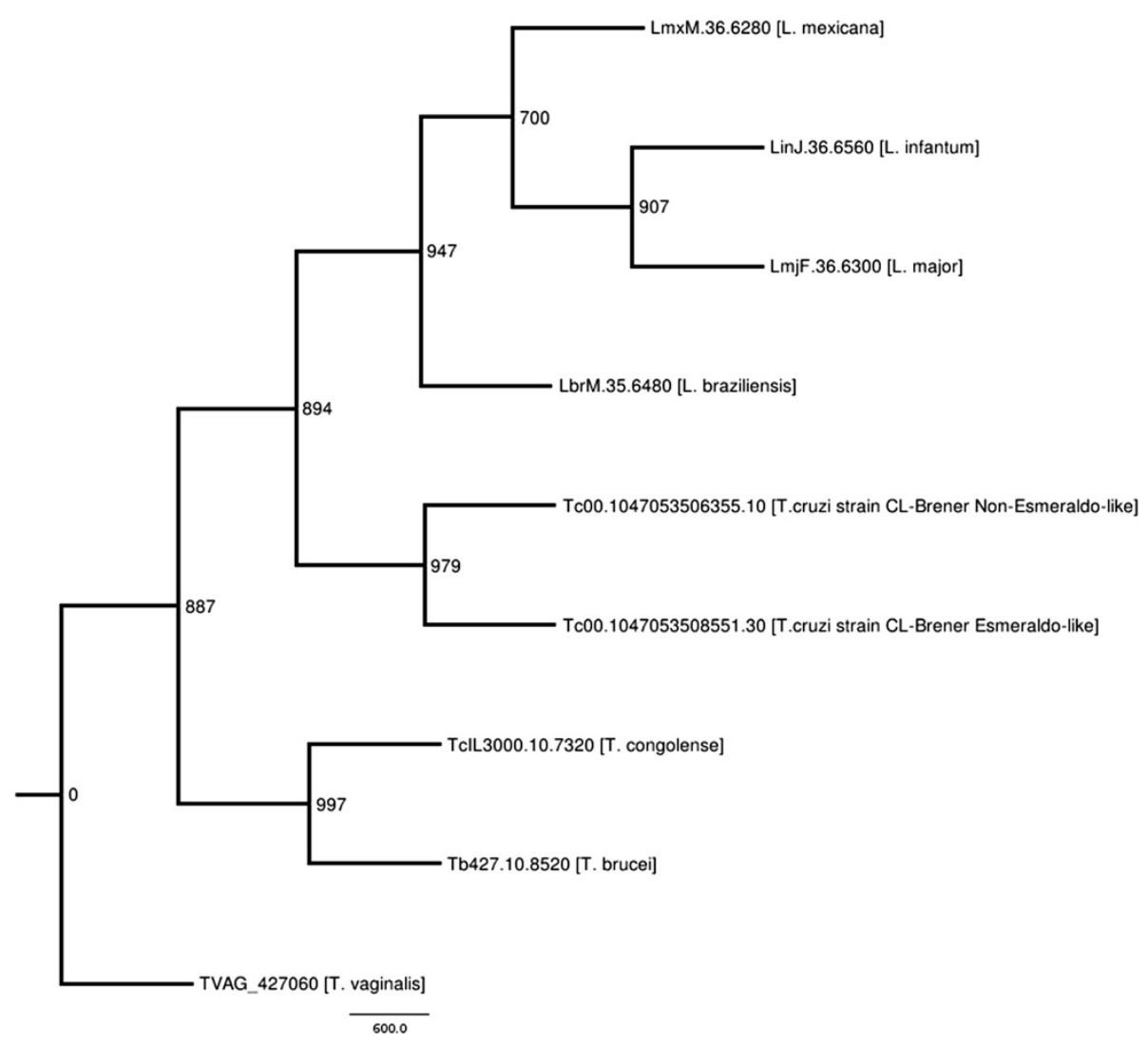

Figure 1 Maximum likelihood phylogenetic tree based on sequences of hexose transporters of Trypanosoma cruzi and other organisms. In the consensus bootstrap tree (1000 replicates) shown, the numbers above each branch represent the bootstrap confidence percentage and the GenBank accession numbers are provided for each species included in the tree. 

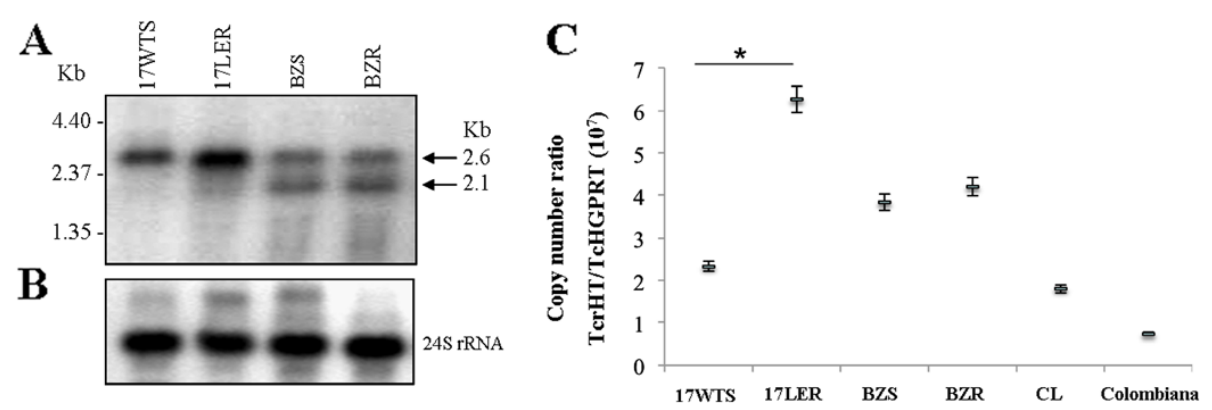

Figure 2 Levels of transcription of the $T c r H T$ gene in BZ resistant and susceptible $T$. cruzi populations and strains showing: (A) Northern blot profiles of total RNA extracts obtained using a ${ }^{32} \mathrm{P}$-labeled TcrHT-specific probe; (B) the quantitative control in which the same membrane was exposed to a T. cruzi 24 S ribosomal RNA probe; and (C) levels of TcrHT mRNA as determined quantitatively (relative to the single-copy housekeeping gene TCHGPRT) by real-time RT-PCR. Mean values of the copy number ratio TcrHT/TCHGPRT \pm standard deviations from three independent experiments are indicated. The mean values for 17 WTS and 17 LER are significantly different $\left({ }^{*} p<0.001\right)$, whilst the BZR versus BZS and the CL versus Colombiana mean values show no difference.

than in the susceptible 17 WTS population. In contrast, no differences in mRNA TcrHT levels for both transcripts were detected between the BZR and BZS populations. The results obtained from the quantitative real-time RTPCR analyses confirmed these findings and indicated that the number of TcrHT cDNA molecules was 2.6-fold higher in the 17 LER population than in the 17 WTS population, while there were no differences in TcrHT transcription levels between populations BZR and BZS or between the naturally-resistant strain Colombiana and the susceptible strain CL (Figure 2C).

\section{Genomic organization and copy number of the TcrHT gene}

Southern blot assays were carried out using samples of T. cruzi genomic DNA that had been restricted with the endonucleases SalI, which has one restriction site within TcrHT, and EcoRI, which has no restriction site within the reference TcrHT sequence (U05588). Hybridization of the blots of SalI-digested DNA against a TcrHTspecific probe revealed a major band of $3.2 \mathrm{~kb}$ and another less intense band of $2.8 \mathrm{~Kb}$ for all $T$. cruzi samples analyzed (Figure 3A). In addition, the result showed two bands of approximately 6.5 and $9.0 \mathrm{~kb}$ in populations 17 WTS and 17 LER from T. cruzi group I and two bands of 8.0 and $9.5 \mathrm{~kb}$ in populations BZR, BZS and clones $4 \mathrm{~S}$ and 16R, from T. cruzi group II (Figure 3A). Southern blots of DNA that had been digested with EcoRI showed one major band of $2.8 \mathrm{~kb}$ and three bands of approximately 4.0, 6.5 and $9.0 \mathrm{~kb}$ in populations 17 WTS and 17 LER from T. cruzi group I, and two bands of 11.0 and $12.0 \mathrm{~kb}$ in populations BZR, BZS and clones $16 \mathrm{R}$ and $4 \mathrm{~S}$, from $T$. cruzi group II (Figure $3 \mathrm{~B}$ ). The band profiles indicated that TcrHT is a multicopy gene, thus confirming the findings of the in silico analyses. The Southern blot assays also revealed that TcrHT exhibits

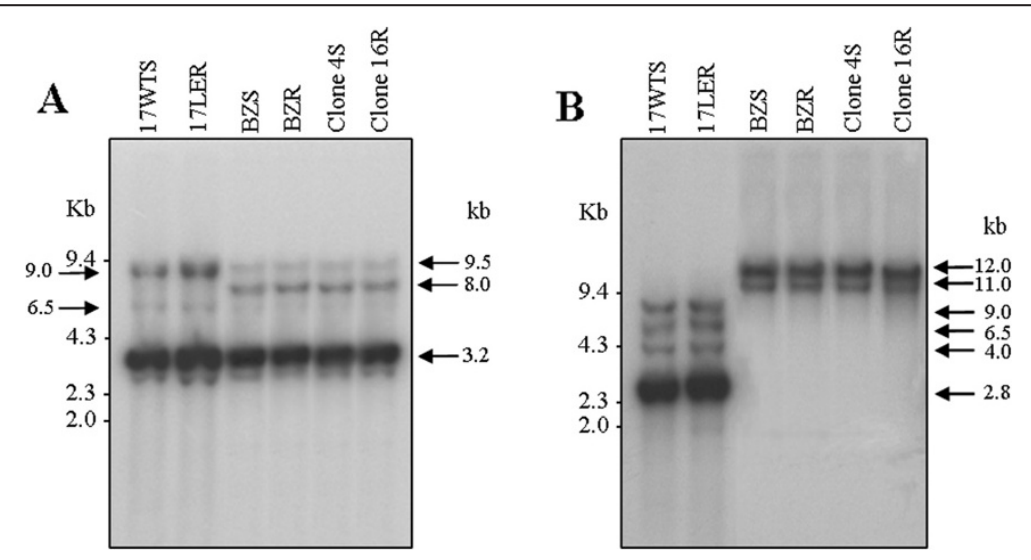

Figure 3 Southern blot analyses of the TcrHT gene from BZ resistant and susceptible $T$. cruzi populations and strains. Preparations of genomic DNA $(14 \mu \mathrm{g})$ were digested with endonucleases Sall $(\mathbf{A})$ or EcoRI $(\mathbf{B})$, separated by electrophoresis on a $1 \%$ agarose gel and hybridized with a ${ }^{32}$ P-labeled TcrHT-specific probe. The molecular weight markers were derived from $\lambda$ phage DNA digested with Hindlll and $\varphi$ X 174 DNA digested with Hae III. 
group-specific polymorphism in that there are SalI and EcoRI restriction sites in the gene sequence present in strains from $T$. cruzi group I that are absent in the gene sequence present in strains from $T$. cruzi group II. Densitometric analyses of the blots showed that the intensities of the bands were similar in all of the T. cruzi samples analyzed, signifying that TcrHT is not amplified in the genome of $T$. cruzi resistant populations.

\section{Chromosomal location of the TcrHT gene}

The profiles obtained following PFGE separation of the chromosomes of resistant and susceptible T. cruzi strains are presented in Figure 4A. Southern blot analysis of these chromosomes with a $\mathrm{Tc} H \mathrm{H}$-specific probe revealed a marked heterogeneity in chromosome size (Figure 4B), and showed that the gene is located on chromosomes of ca. 1.85 and $2.02 \mathrm{Mb}$ in T. cruzi group I (17 LER, 17 WTS and Yuyu) and of 2.21 and $2.6 \mathrm{Mb}$ in T. cruzi group II (BZR, BZS and clones 16R and 4 S). However, the profile of SC-28, which is also classified as T. cruzi group I, was clearly strain-specific with TcrHT located on chromosomes of approximately 2.0 and 2.4 Mb. Comparison of the chromosomal bands recognized by the TcrHT probe indicated that there were no differences between resistant and susceptible populations of T. cruzi. This finding is consistent with the hypothesis that TcrHT is not amplified in the genome of the resistant strains of the parasite analyzed.

\section{Activity of TcrHT in T. cruzi populations}

Figure 5 displays the results of the HT activity assays represented as the amount of glucose (mol) taken up by the cells / total amount of protein $(\mathrm{mg})$ at different time intervals. With the exception of the resistant population 17 LER, the efficiencies of glucose transport were very similar for all T. cruzi samples analyzed at all time intervals studied. Factorial ANOVA tests revealed that the reduced rate of glucose transport observed for population 17 LER in comparison with the other T. cruzi populations and strains was statistically significant $(\mathrm{F}=90.12$ and $\mathrm{p}<0.001)$. Considering each resistant/susceptible pair separately, the amount of glucose transported by 17 LER cells was, on average, $40 \%$ less than that transported by 17 WTS cells at all time intervals analyzed. Factorial ANOVA tests showed that this difference was also statistically significant $(F=1.148$ and $p<0.001)$. No differences in glucose transport were observed between pairs BZR and BZS $(\mathrm{p}=0.072)$ and between strains Colombiana and CL $(\mathrm{p}=0.5)$.

\section{Sequencing data}

DNA sequencing of TcrHT gene from T. cruzi susceptible and resistant strains was performed in order to investigate whether point mutations could be causing difference in glucose uptake. Multi-alignment of the TcrHT nucleotide and amino acid sequences revealed a total of 31 nucleotide mutations (data not shown) leading to the substitution of 11 amino acid residues (Figure 6). We found no association between the nucleotide mutations or amino acid substitutions and the resistance phenotype. In contrast, we found that all 28 nucleotide mutations and 9 of the amino acid substitutions were $T$. cruzi strain-specific. Our analysis revealed that the nucleotide and amino acid sequences of the TcrHT from 17WTS and 17LER were identical. This result suggests that the difference in glucose transport between 17WTS and 17LER populations is not due to point mutations, but probably to differences in the TcrHT protein expression level.

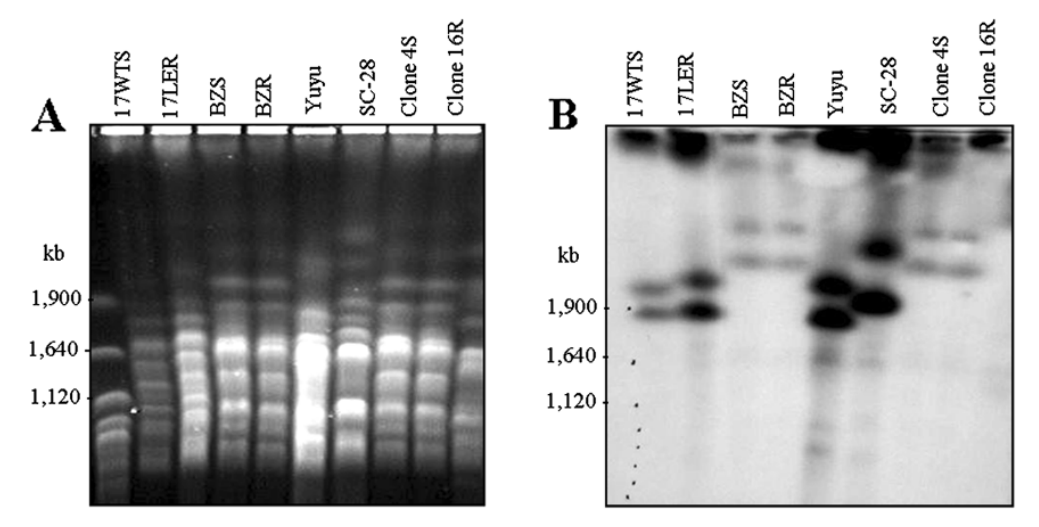

Figure 4 Chromosomal locations of theTcrHT gene in BZ resistant and susceptible $T$. cruzi populations and strains showing: (A) chromosomal bands that had been separated by pulsed-field gel electrophoresis and stained with ethidium bromide; and (B) Southern blots obtained by hybridizing the chromosomal bands with a ${ }^{32}$ P-labeled TcrHT-specific probe. Whole chromosomes from Saccharomyces cerevisiae were employed as molecular weight markers. 


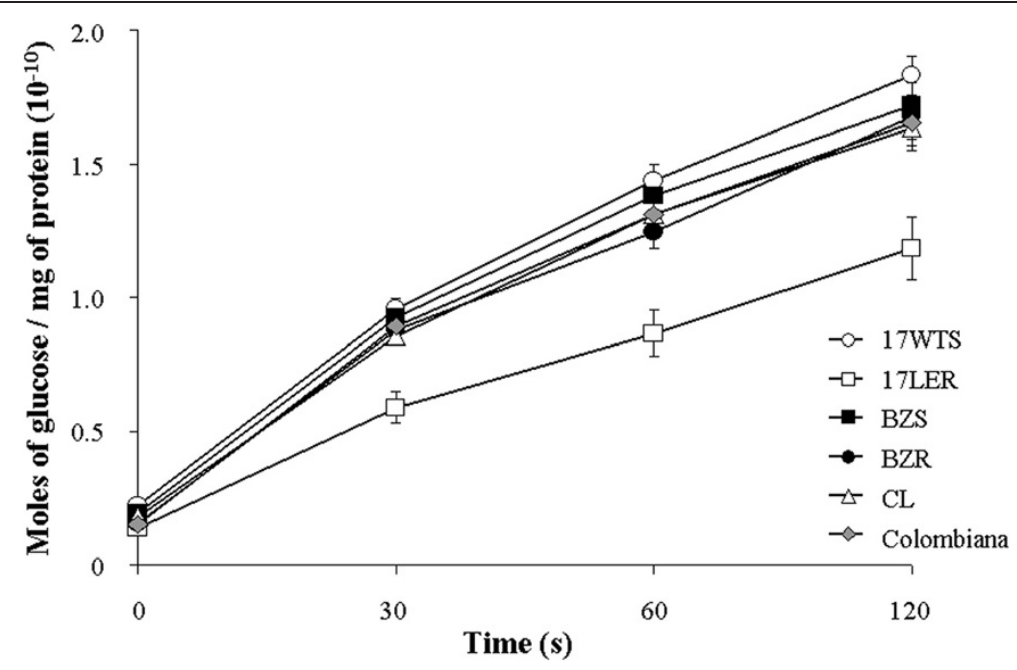

Figure 5 Comparison of hexose transporter activities among BZ resistant and susceptible $T$. cruzi populations and strains showing the quantities of 6 -deoxy-D-[6- $\left.{ }^{3} \mathrm{H}\right]$ glucose transported per $\mathrm{mg}$ of total protein as a function of time. The values shown represent the means of three independent experiments and the error bars indicate the standard error of the mean ( \pm SEM). The mean values for 17 WTS and 17 LER are significantly different $(p<0.001)$, whilst the BZR versus BZS and the Colombiana versus $C L$ mean values show no such differences $(p=0.072$ and $p=0.5$, respectively).

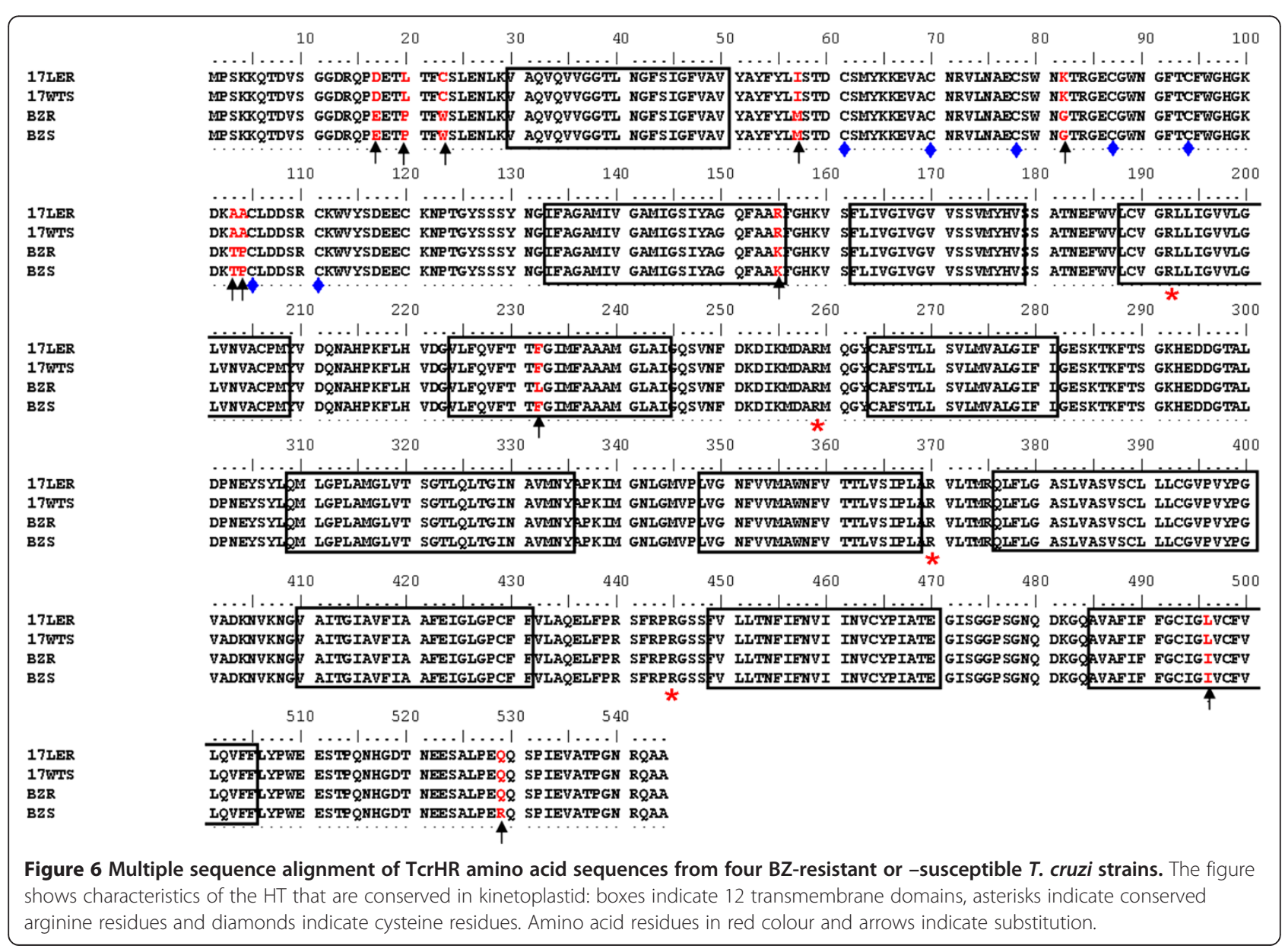




\section{Discussion}

Hexose transporter proteins are considered to be potential targets for the development of therapies against parasitic diseases since they could be inhibited, thus disrupting the entry of vital nutrients into the parasite, or employed as transporters of toxic compounds [15]. In the present study, the gene encoding HT has been characterized in populations and strains of $T$. cruzi that are resistant or susceptible to BZ. The ORF of TcrHT is $1,635 \mathrm{bp}$ in length and encodes for a protein of 544 amino acids with a predicted mass of $60 \mathrm{kDa}$. As is characteristic of HTs belonging to the GLUT1 super family of facilitative glucose transporters, the protein presented 12 conserved transmembrane domains. Phylogenetic analyses showed that the amino acid sequence of TcrHT is closely related (with identities of around 60\%) to that of other species of trypanosomatids. Much lower identities $(<25 \%)$ were observed between TcrHT and HT sequences from mammals. One important prerequisite in the selection of a potential target for chemotherapy against a parasitic disease is that the target be absent, or suitably distinct from its homologue, in the mammalian host [34]. According to the data presented herein, it is likely that a drug acting via TcrHT would be able to affect the parasite without disrupting the metabolism of the vertebrate host.

In silico and Southern blot analyses revealed that the profile of TcrHT was consistent with a multicopy gene and showed nucleotide sequence polymorphism related to T. cruzi group. A total of 15 sequences related to transporter protein were present in the $T$. cruzi database [24]. Among these sequences, nine sequences were annotated as hexose/sugar transporter protein being four complete copies, three incomplete and two truncated. The remaining six sequences were annotated as hypothetical proteins, however, they present the motif of "putative transporter protein". Tetaud et al. [12] had previously determined by partial digestion of genomic DNA that at least eight copies of TcrHT were present in tandem array. More recently, Arner et al. [33] developed an in silico approach involving multiple alignment of any $T$. cruzi sequence against all sequences generated by the genome project for this parasite and estimated that the glucose transporter gene is present in 35 copies in the diploid genome. The authors have chosen to treat the two allelic copies of a gene as two gene copies. Then, if we consider that each TcrHT gene is represented by two allelic forms, there are approximately 17 copies of this gene in the genome of the T. cruzi CL Brener. This value is very close to that established in the present study, and is supported by Southern blot analyses showing that TcrHT exhibits a profile that is typical of a multicopy gene. The present study has revealed that TcrHT is not amplified in the genome of any of the T. cruzi resistant populations or strains investigated. Interestingly, however, the TrrHT gene exhibits nucleotide polymorphism among the different strains analyzed, which is associated with T. cruzi belonging to groups I or II. Moreover, the TcrHT gene was found to be located in two chromosomes in all of the resistant and susceptible $T$. cruzi strains analyzed. In the T. cruzi chromosomal assemble [35], the TrrHT gene is also located in two chromosomes, namely, the TcChr37-S and TcChr26-S from the Esmeraldo-like haplotype, and the TcChr37-P and TcChr26-P from the non-Esmeraldo like haplotype. The remaining sequences annotated as hypothetical protein (putative transporter protein) are located on chromosome TcChr13 and TcChr39 of both CL Brener haplotypes. Clearly, chromosomal location of TcrHT is not related to the drug resistance phenotype but is associated with the $T$. cruzi group of the strain analyzed with the single exception of strain SC-28, which presented a particular chromosomal pattern.

In the present study, Northern blot analyses of TcrHT revealed one transcript of $2.6 \mathrm{~kb}$ for the population classified as T. cruzi group I and two transcripts of 2.1 and $2.6 \mathrm{~kb}$ for the populations classified as T. cruzi group II. This finding is in accord with that of Tetaud et al. [12] who detected transcripts of 2.1 and $2.6 \mathrm{~kb}$ in the T. cruzi group II strain. According to these authors, the two transcripts encode for the same protein and the size difference is due to variations in the 3' untranslated region. It is known that specific sequences in this untranslated region are capable of regulating gene expression [36,37], since they permit the interaction with factors that stimulate or inhibit the transcription process according to the developmental stage of the parasite.

Northern blot and real time-PCR analyses revealed that the expression of $\mathrm{TcrHT}$ was 2.6-fold greater in the resistant population 17 LER than in its susceptible counterpart 17 WTS. Different attempts to produce polyclonal antibody against the TcrHT recombinant protein failed, then unfortunately the protein expression level could not be analyzed in these T. cruzi samples. As described in the literature the hexose transporter activity is an important approach to verify the involvement of this transporter in the drug resistance phenotype. Then, we analyzed the amount of glucose taken up by the cells / total amount of protein at different time intervals and verified that the hexose transporter activity was $40 \%$ lower in the 17 LER population than in all other T. cruzi samples analyzed. These results could reflect a decrease of TcrHT protein expression level in the BZ-resistant population 17LER or these parasites could be expressing a TcrHT isoform with lower affinity for glucose. Subsequently, we analysed polymorphisms in the TcrHT nucleotide and amino acid sequences. Our analysis revealed that the TcrHT sequences from 17WTS and 17LER are 
identical. This result suggests that the difference in glucose transport between 17WTS and 17LER populations is not due to point mutations, but probably to differences in the TcrHT protein expression level. Since the regulation of trypanosomatid gene expression occurs mainly at the post-transcriptional level [38], maybe the mRNA of the TcrHT gene in the resistant parasites may not be efficiently translated, thus presenting lower protein expression that could reflect in the lower hexose activity. The discrepancy between increased HT mRNA level and decreased glucose transport can not only be explained by decreased protein expression but also by decreased transport of the protein to the plasma membrane or increased endocytosis of the transporter.

The regulation of the stability and translation of mRNA plays a major role in modulating gene expression in T. cruzi [39]. In addition to mRNA stability, some elements, consisting of AU-rich sequences improve translational efficiency by interaction with specific RNA binding proteins (RBP) $[40,41]$. In agreement with our results, an inverse correlation between mRNA and protein levels of some genes in T. cruzi has also been previously reported in the literature. Silva et al. [42] showed that $\alpha$ and $\beta$ tubulin mRNAs are three to six-fold more abundant in epimastigote forms than in trypomastigote and amastigote forms of $T$. cruzi. However, the protein levels of free $\alpha$ and $\beta$ tubulin subunits are more abundant in trypomastigotes and amastigotes than in epimastigotes. Metacyclogenin gene (TcMet) also suffers posttranscriptional regulation, since a similar pattern of TcMet mRNA expression is observed in both replicating and differentiating epimastigote forms of $T$. cruzi. However, the TcMet protein was detected only in differentiating epimastigotes [43]. Previous data obtained by our group, also describes differences between mRNA and protein levels of some T. cruzi genes. Although the levels of TcTAT (tyrosine aminotransferase) and TcHSP-70 (heat shock protein $70 \mathrm{kDa}$ ) mRNAs were higher in the T. cruzi BZ-resistant population 17LER, no corresponding increases were observed in the levels of TcTAT and TcHSP-70 protein expression [11,44].

It is known that the over-expression of members of the ATP-binding cassette (ABC) superfamily of membrane transporters can confer multidrug resistance in a variety of organisms. However, Nourani et al. [45] have shown that an ABC transporter knockout mutant of Saccharomyces cerevisiae maintained the multidrug resistance phenotype and also presented decreased expression of the hexose transporters HTX9 and HTX11. This finding led to the suggestion that these transporters might afford a route for drug entrance and that their downregulation would favor the survival of the yeast in the presence of the drug. In support of this hypothesis, Uzcategui et al. [46] reported a decrease of $80 \%$ in glucose transport in a strain of L. amazonensis resistant to glibenclamide (GLIB), an inhibitor of $A B C$ transporters, while Machuca et al. [47] showed that glucose accumulation in a GLIB-resistant strain of this parasite was 4.5 -fold slower than that of the susceptible parental strain. Decreased glucose accumulation has also been observed in GLIB-resistant cell lines of L. major [47]. The results of the present study suggest that the BZ resistant population 17 LER presents a decreasing glucose uptake in response to drug pressure, and that this could favor the survival of the parasite in the presence of BZ since less toxic compounds would enter into the cell.

Many of studies concerning drug resistance mechanisms in parasites were based on models produced by artificial induction of resistance. In contrast, there is very little information available on the biochemical mechanisms underlying drug resistance in field isolates. The data presented in this paper show that the hexose transporter TcrHT has a decreased degree of activity in the in vitro induced BZ resistant T. cruzi population, a situation that is different from that observed in the in vivo selected resistant line. In agreement with our results, Villareal et al. [48] observed that the mechanisms associated with natural resistance to drugs differ from those in induced resistance. The mechanism of drug resistance, such as that to BZ, is often complex and typically ensues as a result of the concomitant activation of multiple, often overlapping, signalling pathways, including factors associated with the host immune system, which may enhance the susceptibility of the parasite to the drug [49].

\section{Conclusion}

As part of an on-going project dedicated to the elucidation of the molecular basis of drug resistance in T. cruzi, we have studied a selection of genes that are differentially expressed in BZ resistant and susceptible populations of the parasite $[30,31,50]$. The results obtained suggest that the mechanisms by which resistant strains of $T$. cruzi evade the effects of the drug are somewhat complex, and highlight the necessity to perform functional analyses of TcrHT in order to confirm our hypothesis that TcrHT is involved in the drug resistance phenotype. However, TcrHT can still be considered as a potential therapeutic target since it constitutes the point of uptake of essential nutrients for the parasite. A decrease in the influx of hexoses can compromise parasite infection, since these sugars are essential for ATP production and for the biosynthesis of glycoconjugates that are secreted or expressed on trypanosomatid membranes [51] and are directly related to virulence and the ability of the parasite to escape from the immune system of the host. 


\section{Additional file}

Additional file 1 Table S1: Analysis of the TcrHT gene in the genomic assembly of Trypanosoma cruzi. Figure S1. Multiple sequence alignment of TcrHT amino acid complete sequences annotated in the $T$. cruzi genome assembles. The figure shows characteristics of the $\mathrm{HT}$ that are conserved in kinetoplastid: boxes indicate 12 transmembrane domains, asterisks indicate conserved arginine residues and diamonds indicate cysteine residues. Amino acid residues in colored indicate substitution.

\section{Competing interests}

The authors declare that they have no competing interests.

\section{Authors' contributions}

Conceived and designed the experiments: PFS, AJR, SMFM; performed the experiments: PFS, DSM, AMR, ELF, RPPS, SMFM; analyzed the data: PFS, JCR, AMR, ELF, DSM, RPPS, GO, AJR, SMFM; helped to draft the manuscript: PFS, JCR, DSM, AMR, ELF, RPPS, GO, AJR, SMFM. All authors read and approved the final manuscript.

\section{Acknowledgements}

The authors are grateful to the Program for Technological Development in Tools for Health-PDTIS-FIOCRUZ for the use of facilities and MSc. Aline Daiane Schlindwein (Laboratory of Protozoology, Federal University of Santa Catarina - UFSC) for sequencing the T. cruzi DNA samples. Financial support for the study was received from the following Brazilian agencies: Conselho Nacional de Desenvolvimento Científico e Tecnológico (CNPq), Fundação de Amparo à Pesquisa do Estado de Minas Gerais (FAPEMIG), Coordenação de Aperfeiçoamento de Pessoal de Nível Superior (CAPES) and Programa de Apoio à Pesquisa Estratégica (PAPES - FIOCRUZ). PFS and GO were supported by the National Institute of Health (TW007012), while SMFM and GO received grants from FAPEMIG (PPM-00536-11 and PPM-00439-10, respectively). DSM received FIOCRUZ Fellow and AMR and ELF are CNPq PhD student Fellows. RPPS, GO, AJR and SMFM are CNPq Research Fellows.

\section{Author details}

${ }^{1}$ Centro de Pesquisas René Rachou/FIOCRUZ, Avenida Augusto de Lima 1715, Belo Horizonte 30190-002, MG, Brazil. ${ }^{2}$ Centro de Excelência em Bioinformática, FIOCRUZ, Rua Araguari 741, Belo Horizonte 30190-110, MG, Brazil. ${ }^{3}$ Instituto de Ciências Biológicas, Universidade Federal de Minas Gerais, Belo Horizonte 31270-910, MG, Brazil.

Received: 26 March 2012 Accepted: 2 August 2012

Published: 7 August 2012

\section{References}

1. World Health Organization: Chagas disease (American trypanosomiasis), Fact sheet N³40. 2010. Available from: http://www.who.int/mediacentre/ factsheets/fs340/en/index.html (Accessed June 2011).

2. Cançado JR: Long term evaluation of etiological treatment of Chagas disease with benznidazole. Rev Inst Med Trop São Paulo 2002, 44:29-37.

3. Pérez-Molina JA, Perez-Ayala A, Moreno S, Fernandez-Gonzalez MC, Zamora J, Lopez-Velez R: Use of benznidazole to treat chronic Chagas' disease: a systematic review with a meta-analysis. J Antimicrob Chemother 2009, 64:1139-1147.

4. Viotti R, Vigliano C, Lococo B, Alvarez MG, Petti M, Bertocchi G, et al: Side effects of benznidazole as treatment in chronic Chagas disease: fears and realities. Expert Rev Anti Infect Ther 2009, 7:157-163.

5. Brener Z, Costa CA, Chiari C: Differences in the susceptibility of Trypanosoma cruzi strains to active chemotherapeutic agents. Rev Inst Med Trop São Paulo 1976, 18:450-455.

6. Filardi LS, Brener Z: Susceptibility and natural resistance of Trypanosoma cruzistrains to drugs used clinically in Chagas disease. Trans $R$ Soc Trop Med Hyg 1987, 81:755-759.

7. Murta SM, Gazzinelli RT, Brener Z, Romanha AJ: Molecular characterization of susceptible and naturally resistant strains of Trypanosoma cruzi to benznidazole and nifurtimox. Mol Biochem Parasitol 1998, 93:203-214.

8. Toledo MJ, Bahia MT, Veloso VM, Carneiro CM, Machado-Coelho GL, Alves $\mathrm{CF}$, et al: Effects of specific treatment on parasitological and histopathological parameters in mice infected with different Trypanosoma cruzi clonal genotypes. J Antimicrob Chemother 2004 53:1045-1053.

9. Wilkinson SR, Taylor MC, Horn D, Kelly JM, Cheeseman I: A mechanism for cross-resistance to nifurtimox and benznidazole in trypanosomes. Proc Natl Acad Sci USA 2008, 105:5022-5027.

10. Brener $Z$, Andrade Z, Barral-Neto M: Tratamento etiológico da doença de Chagas pelo benzonidazol. In Trypanosoma cruzi e Doença de Chagas. 2nd edition. Edited by Brener Z, Andrade Z, Barral-Neto M. Rio de Janeiro: Guanabara Koogan; 2000:389-405.

11. Murta SM, Nogueira FB, Dos Santos PF, Campos FM, Volpe C, Liarte DB, et al: Differential gene expression in Trypanosoma cruzi populations susceptible and resistant to benznidazole. Acta Trop 2008, 107:59-65.

12. Tetaud E, Bringaud F, Chabas S, Barrett MP, Baltz T: Characterization of glucose transport and cloning of a hexose transporter gene in Trypanosoma cruzi. Proc Natl Acad Sci USA 1994, 91:8278-8282.

13. Mueckler M: Facilitative glucose transporters. Eur J Biochem 1994, 219:713-725.

14. Barrett MP, Tetaud E, Seyfang A, Bringaud F, Baltz T: Trypanosome glucose transporters. Mol Biochem Parasitol 1998, 91:195-205.

15. Joët T, Eckstein-Ludwig U, Morin C, Krishna S: Validation of the hexose transporter of Plasmodium falciparum as a novel drug target. Proc Natl Acad Sci USA 2003, 100:7476-7479.

16. Rodriguez-Contreras D, Landfear SM: Metabolic changes in glucose transporter-deficient Leishmania mexicana and parasite virulence. J Biol Chem 2006, 281:20068-20076.

17. Feistel T, Hodson CA, Peyton DH, Landfear SM: An expression system to screen for inhibitors of parasite glucose transporters. Mol Biochem Parasitol 2008, 162:71-76.

18. Tetaud E, Chabas S, Giroud C, Barrett MP, Baltz T: Hexose uptake in Trypanosoma cruzi: structure-activity relationship between substrate and transporter. Biochem J 1996, 317:353-359.

19. Murta SM, Romanha AJ: In vivoselection of a population of Trypanosoma cruzi and clones resistant to benznidazole. Parasitology 1998, 116:165-171.

20. Nirdé $P$, Larroque C, Barnabe C: Drug-resistant epimastigotes of Trypanosoma cruzi and persistence of this phenotype after differentiation into amastigotes. Comp Rend Acad Sci 1995, 318:1239-1244.

21. Zingales B, Andrade SG, Briones MR, Campbell DA, Chiari E, Fernandes O, et al: A new consensus for Trypanosoma cruzi intraspecific nomenclature: second revision meeting recommends Tcl to TcVI. Mem Inst Oswaldo Cruz 2009, 104:1051-1054

22. Camargo EP: Growth and differentiation in Trypanosoma cruzi. I. Origin of metacyclic trypanosomes in liquid media. Rev Inst Med Trop São Paulo 1964, 6:93-100.

23. Finn RD, Clements J, Eddy SR: HMMER web server: interactive sequence similarity searching. Nucleic Acids Res 2011, 39:W29-W37.

24. El-Sayed NM, Myler PJ, Bartholomeu DC, Nilsson D, Aggarwal G, Tran AN, et al: The genome sequence of Trypanosoma cruzi, etiologic agent of Chagas disease. Science 2005, 309:409-415.

25. Katoh K, Misawa K, Kuma K, Miyata T: MAFFT: a novel method for rapid multiple sequence alignment based on fast Fourier transform. Nucleic Acids Res 2002, 30:8.

26. Capella-Gutierrez S, Silla-Martinez JM, Gabaldon T: TrimAl: a tool for automated alignment trimming in large-scale phylogenetic analyses. Bioinformatics 2009, 25:1972-1973.

27. Abascal F, Zardoya R, Posada D: ProtTest: selection of best-fit models of protein evolution. Bioinformatics 2005, 21:2104-2105.

28. Jones DT, Taylor WR, Thornton JM: The rapid generation of mutation data matrices from protein sequences. Comput Appl Biosci 1992, 19928:275-282.

29. Felsenstein J: Phylip. 2009. http://evolution.genetics.washington.edu/phylip. html.

30. Nogueira FB, Krieger MA, Nirde P, Goldenberg S, Romanha AJ, Murta SM: Increased expression of iron-containing superoxide dismutase-A (TcFeSOD-A) enzyme in Trypanosoma cruzi population with in vitro-induced resistance to benznidazole. Acta Trop 2006, 100:119-132.

31. Murta SM, Krieger MA, Montenegro LR, Campos FF, Probst CM, Avila AR, et al: Deletion of copies of the gene encoding old yellow enzyme (TcOYE), a NAD(P)H flavin oxidoreductase, associates with in vitro-induced benznidazole resistance in Trypanosoma cruzi. Mol Biochem Parasitol 2006, 146:151-162.

32. Eisenthal R, Game S, Holman GD: Specificity and kinetics of hexose transport in Trypanosoma brucei. Biochim Biophys Acta 1989, 985:81-89. 
33. Arner E, Kindlund E, Nilsson D, Farzana F, Ferella M, Tammi MT, et al: Database of Trypanosoma cruzi repeated genes: 20,000 additional gene variants. BMC Genomics 2007, 8:391.

34. Myler PJ: Searching the TriTryp genomes for drug targets. Adv Exp Med Biol 2008, 625:133-140.

35. Weatherly DB, Boehlke C, Tarleton RL: Chromosome level assembly of the hybrid Trypanosoma cruzi genome. BMC Genomics 2009, 10:255.

36. Nozaki T, Cross GA: Effects of 3' untranslated and intergenic regions on gene expression in Trypanosoma cruzi. Mol Biochem Parasitol 1995, 75:55-67.

37. Teixeira SM, Kirchhoff LV, Donelson JE: Post-transcriptional elements regulating expression of mRNAs from the amastin/tuzin gene cluster of Trypanosoma cruzi. J Biol Chem 1995, 270:22586-22594.

38. Vanhame L, Pays E: Control of gene expression in Trypanosomes. Microbiol 1995, 59:223-240.

39. Goldenberg S, Avila AR: Aspects of Trypanosoma cruzi stage differentiation. Adv Parasitol 2011, 75:285-305.

40. Di Noia JM, D'Orso I, Sánchez DO, Frasch AC: AU-rich elements in the 3'-untranslated region of a new mucin-type gene family of Trypanosoma cruzi confers mRNA instability and modulates translation efficiency. J Biol Chem 2000, 275:10218-10227.

41. D'Orso I, Frasch AC: TcUBP-1, a developmentally regulated U-rich RNA-binding protein involved in selective mRNA destabilization in trypanosomes. J Biol Chem 2001, 276:34801-34809.

42. da Silva RA, Bartholomeu DC, Teixeira SM: Control mechanisms of tubulin gene expression in Trypanosoma cruzi. Int J Parasitol 2006, 36:87-96.

43. Avila AR, Yamada-Ogatta SF, da Silva Monteiro V, Krieger MA, Nakamura CV, de Souza W, Goldenberg S: Cloning and characterization of the metacyclogenin gene, which is specifically expressed during Trypanosoma cruzi metacyclogenesis. Mol Biochem Parasitol 2001, 117:169-177.

44. Rego JV, Murta SMF, Nirdé P, Nogueira FB, Andrade HM, Romanha AJ: Trypanosoma cruzi: Characterisation of the gene encoding tyrosine aminotransferase in benznidazole-resistant and susceptible populations. Exp Parasitol 2008, 118:111-117.

45. Nourani A, Wesolowski-Louvel M, Delaveau T, Jacq C, Delahodde A: Multiple-drug-resistance phenomenon in the yeast Saccharomyces cerevisiae: involvement of two hexose transporters. Mol Cell Biol 1997, 17:5453-5460.

46. Uzcategui NL, Figarella K, Camacho N, Ponte-Sucre A: Substrate preferences and glucose uptake in glibenclamide-resistant Leishmania parasites. Comp Biochem Physiol C Toxicol Pharmacol 2005, 140:395-402.

47. Machuca C, Rodriguez A, Herrera M, Silva S, Ponte-Sucre A: Leishmania amazonensis: metabolic adaptations induced by resistance to an $A B C$ transporter blocker. Exp Parasitol 2006, 114:1-9.

48. Villarreal D, Nirde P, Hide M, Barnabe C, Tibayrenc M: Differential gene expression in benznidazole-resistant Trypanosoma cruzi parasites. Antimicrob Agents Chemother 2005, 49:2701-2709.

49. Murta SMF, Ropert C, Alves RO, Gazzinelli RT, Romanha AJ: In vivo treatment with benznidazole enhances phagocytosis, parasite destruction and cytokine release by macrophages during infection with a drug-susceptible but not with a derived drug-resistant Trypanosoma cruzi population. Parasite Immunol 1999, 21:535-544.

50. Campos FM, Liarte DB, Mortara RA, Romanha AJ, Murta SM: Characterization of a gene encoding alcohol dehydrogenase in benznidazole-susceptible and -resistant populations of Trypanosoma cruzi. Acta Trop 2009, 111:56-63.

51. Ferguson MA: The structure, biosynthesis and functions of glycosylphosphatidylinositol anchors, and the contributions of trypanosome research. J Cell Sci 1999, 112:2799-2809.

doi:10.1186/1756-3305-5-161

Cite this article as: dos Santos et al: Molecular characterization of the hexose transporter gene in benznidazole resistant and susceptible populations of Trypanosoma cruzi. Parasites \& Vectors 2012 5:161.

\section{Submit your next manuscript to BioMed Central and take full advantage of:}

- Convenient online submission

- Thorough peer review

- No space constraints or color figure charges

- Immediate publication on acceptance

- Inclusion in PubMed, CAS, Scopus and Google Scholar

- Research which is freely available for redistribution

Submit your manuscript at www.biomedcentral.com/submit
C Biomed Central 